\title{
Donor Milk Policies for Level 1 Newborn Care: A Descriptive Analysis
}

\author{
Kaitlin H. Drouin, ${ }^{1}$ Jennifer F. Riley, ${ }^{2}$ Charis Benjamin, ${ }^{1}$ Katherine E. Gregory, ${ }^{1-3}$ \\ Sarbattama Sen, ${ }^{1,3}$ and Mandy B. Belfort ${ }^{1,3}$
}

\begin{abstract}
Background and Objectives: Providing pasteurized donor human milk (DHM) to healthy newborns is an emerging practice. The content of hospital policies that govern this practice is unknown.

Materials and Methods: We collected policies from 15 Northeast U.S. hospitals through (1) a 2017 survey on DHM use and (2) an e-mail listserv of levels 1 and 2 newborn care staff maintained by a regional milk bank. Two authors reviewed each policy and identified how they addressed three predetermined themes: who is eligible to receive DHM, how DHM is used and described, and how lactation is supported. Responses were compared, discussed, and reconciled. Level 1 newborn care was defined as basic care for healthy newborns $\geq 35$ weeks' gestation.

Results: Thirteen of 15 policies stated criteria for DHM eligibility, most commonly as a bridge until mother's supply comes in $(73 \%)$ or for infant medical conditions (67\%). All required consent for DHM. Most did not limit number of days infants could receive DHM (60\%). Nine specified that DHM be discarded 24 hours after thaw, whereas five recommended discarding at 48 hours. Although many (53\%) policies endorsed human milk as the preferred diet for newborns, only $27 \%$ specifically endorsed DHM as the preferred supplementation type. Parent education (73\%) was emphasized, but few (27\%) discussed the importance of establishing mother's milk supply.

Conclusions: Many DHM policies address eligibility criteria for receiving DHM and show how to provide DHM, but few address how to support lactation while DHM is provided, which may be crucial for optimizing long-term breastfeeding outcomes.
\end{abstract}

Keywords: donor milk, supplementation, hospital practices

\section{Introduction}

$\mathbf{R}$ OBUST EVIDENCE SUPPORTS the benefits of breastfeeding for mothers and infants. ${ }^{1}$ Mother's own milk is the recommended diet for newborns and has particular benefits for infants born preterm. ${ }^{2,3}$ When mother's own milk is unavailable to preterm infants, pasteurized donor human milk (PDHM) is the recommended alternative. ${ }^{4}$ For this reason, DHM is used increasingly in level 3 neonatal intensive care units (NICUs). ${ }^{5}$

Our recent study demonstrates that DHM use is not limited to preterm infants in NICUs but in fact is also on the rise in level 1 newborn care units. ${ }^{6}$ Both the Baby-Friendly Hospital Initiative $^{7}$ and The Joint Commission ${ }^{8}$ track exclusive breast milk feeding during the entire newborn hospitalization based on evidence that formula use in newborns may interfere with the establishment of breastfeeding. ${ }^{9}$ The practice of supplementing with DHM can reduce formula use during the hospitalization and thereby improve hospital compliance with Baby Friendly and Joint Commission metrics.

As DHM use in level 1 newborn care broadens, each hospital develops or adapts their policies and guidelines to inform the use of DHM. Although some sources recommend including specific topics, ${ }^{10,11}$ the content of these policies is largely unknown. The purpose of this study was to describe the contents of policies for DHM use in the level 1 newborn care setting in the northeast United States. Specifically, we aimed to determine (1) who is eligible to receive DHM, (2)

\footnotetext{
${ }^{1}$ Department of Pediatric Newborn Medicine, Brigham and Women's Hospital, Boston, Massachusetts.

${ }^{2}$ Department of Nursing, Brigham and Women's Hospital, Boston, Massachusetts.

${ }^{3}$ Harvard Medical School, Boston, Massachusetts.
} 
how DHM is used and how it is described in the policy, and (3) how mothers are supported in establishing a milk supply when supplementation is required.

\section{Materials and Methods}

\section{Design, setting, and sample}

Two authors (K.D. and J.R.) collected policies from hospitals in the northeast United States that reported DHM use in their level 1 newborn care unit. The policies were collected from two sources: a 2017 hospital survey on donor milk use ${ }^{6}$ completed by perinatal hospital staff and by e-mail request to a combined listserv of levels 1 and 2 newborn care staff maintained by Mother's Milk Bank Northeast. Of the 100 birth hospitals contacted for the original survey on DHM through Research Electronic Data Capture (REDCap), 71 responded, of which 23 reported providing DHM in level 1 care; 11 of those uploaded a DHM policy with their response. To increase the number of policies, we subsequently sent an e-mail to the milk bank listserv (95 individuals) and obtained DHM policies from the staff of 4 additional hospitals. We did not receive refusals to these requests for policies, any "missed" policies were due to no response. Level 1 newborn care was defined as a unit providing basic care for healthy newborns $\geq 35$ weeks' gestation. $^{12}$

\section{Measurement}

We identified broad areas of focus, guided by the policy content categories suggested by two sources-an article describing the implementation of a level 1 DHM program at Boston Medical Center, ${ }^{10}$ and a guide for implementing a DHM program published by Mothers' Milk Bank Northeast. ${ }^{11}$ These areas of focus included (1) eligibility criteria, (2) informed consent, (3) parent education, (4) staff education, and (5) the ordering and handling of DHM. We also evaluated how DHM was described in each policy, including its source, safety, and claims of benefits. To capture eligibility criteria, the authors drew on criteria commonly specified for formula supplementation of breastfed newborns in level 1 care, ${ }^{10,13,14}$ and additionally recorded other criteria listed in policies. We avoided drawing inferences from policy content and considered only explicit statements in our analysis. Six policy documents included appendices or attachments (e.g., educational handouts or consent forms) that may have contained information related to our analysis; their content was not analyzed because they represented separate documents external to the policies themselves. Eight hospitals provided up to level 3 or 4 care to newborns and had combined policy documents for DHM use that covered all levels of care. In these cases, we carefully considered whether specific content within the policy applied to newborns receiving level 1 care.

\section{Data collection and analysis}

Policies were reviewed independently by authors K.D. and J.R. A standardized data collection instrument was created prospectively to capture the responses from the reviews of each policy document using REDCap ${ }^{\mathrm{TM}}$, a secure web-based application specifically designed to support data capture and management for research studies. ${ }^{15}$ The instrument contained three types of fields- "yes or no," "check all that apply," and "free text." Free text fields were used to identify areas of the policy that corresponded to the responses in the "yes or no" and "check all that apply" fields for reference. The reviewing authors then compared "yes or no" and "check all that apply" responses for each policy using the REDCap Data Comparison Tool, which identifies and displays differing responses. Inter-rater agreement between K.D. and J.R. was $80 \%$ for the 41 questions contained in the data collection tool. Differing responses were discussed and then reconciled by the two reviewers, and final responses were compiled and analyzed. Descriptive statistics were generated to summarize findings, and excerpts from the free text fields were retained to provide examples and context; qualitative analysis was not performed.

\section{Results}

\section{Eligibility}

Of the 15 policies analyzed, most policies explicitly stated criteria for DHM eligibility $(n=13,87 \%)$ - categories are shown in Figure 1. DHM was commonly allowed in cases of medical conditions affecting the infant, most often hypoglycemia and/or hyperbilirubinemia $(n=8,53 \%)$ (Table 1). It was uncommon for criteria to include medical conditions affecting the mother, or in cases of maternal medication contraindicated with providing breast milk. Eleven policies stated criteria related to mother's supply, restricting DHM use to mothers exclusively breastfeeding and/or as a bridge until mother's milk supply is fully established. Four policies did not state any restrictions based on mother's supply, and only three stated eligibility in cases wherein mothers were unable to pump or breastfeed. Although most policies emphasized that DHM was for short-term use $(n=12)$, few limited the number of days an infant could receive $\operatorname{DHM}(n=6)$. Of those who did, four described a 7-day limit, and two only allowed DHM to be provided up to 5 days. None specified limits by volume.

\section{Consent}

All policies required consent for DHM use, and most $(n=13,87 \%)$ required consent in written form (Table 2).

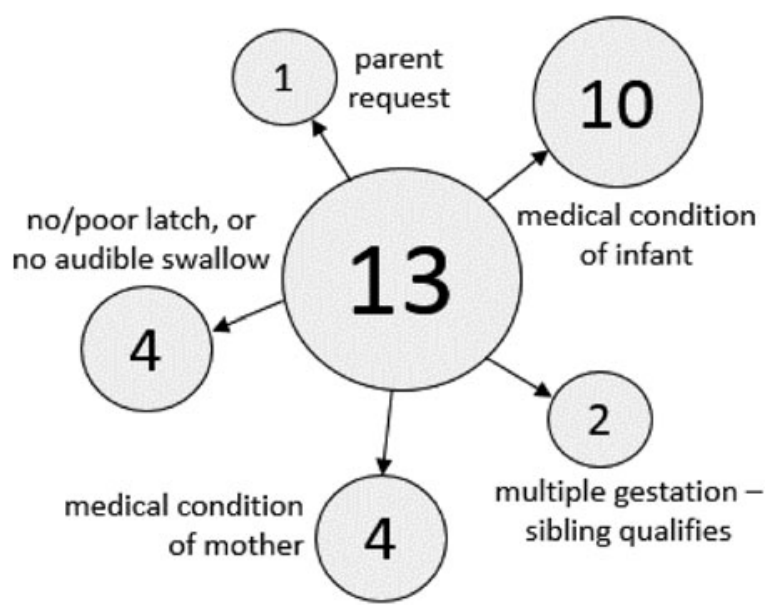

FIG. 1. Level 1 eligibility criteria for donor human milk. 
Table 1. Medical Eligibility CRiteria-Level 1 Donor Human Milk Use $(N=13)$

\begin{tabular}{lr}
\hline & $\mathrm{n}(\%)$ \\
\hline Infant & $10(67)$ \\
Hypoglycemia & $8(53)$ \\
Hyperbilirubinemia with phototherapy & $8(53)$ \\
Excessive weight loss & $5(33)$ \\
Born below a specified birth weight & $3(20)$ \\
Born below specified gestational age & $2(13)$ \\
Inadequate output/hydration & $1(7)$ \\
Mother & $4(27)$ \\
Maternal medication & $3(20)$ \\
Breast infection & $1(7)$ \\
History of breast surgery & $1(7)$ \\
Chronic infection & $1(7)$ \\
HIV & $1(7)$ \\
Nipple pain & $1(7)$ \\
Diabetes & $1(7)$ \\
\hline
\end{tabular}

HIV, human immunodeficiency virus.

Twelve of the 15 policies specified the person(s) able to obtain consent, and most did not require the consenting staff to be a physician. The majority of ordering staff $(n=14,93 \%)$ were neonatal nurse practitioners (NNPs), MDs, physicians, or nonspecific staff.

\section{Parent and staff education}

Policy content related to education and support for parents is given in Table 3. Importance of establishing a milk supply (27\%) and instructions on helping mothers establish their milk supply (20\%) were not commonly included in policies. Policies with these instructions all emphasized evaluating "mother's ability to produce her own milk supply" and making sure "that she has the proper resources (i.e., hospitalgrade electric pump along with instructions on how to establish a milk supply)." One (7\%) policy included the recommendation that mothers of infants receiving DHM be assessed each shift for milk production. No policies suggested DHM be used as part of other breastfeeding interventions such as skin-to-skin, but several (53\%) referenced their hospital's breastfeeding policy. Verbal or written education material for parents was described in most policies (73\%). Some also provided instructions for discharging

Table 2. Donor Human Milk Consent AND ORDERING

\begin{tabular}{lc}
\hline & $\mathrm{n}(\%)$ \\
\hline Consent required & $15(100)$ \\
Written & $13(86)$ \\
Written or verbal & $1(7)$ \\
Unspecified & $1(7)$ \\
Consenting staff specified & $12(80)$ \\
Nonphysician can consent & $11(73)$ \\
Physician required & $1(7)$ \\
Ordering staff specified & $15(100)$ \\
RN & $1(7)$ \\
NNP, MD, physician & $8(53)$ \\
Nonspecific (e.g., "prescriber") & $7(47)$ \\
\hline
\end{tabular}

NNP, neonatal nurse practitioner.
Table 3. Parent Education and Support

\begin{tabular}{lr}
\hline & $\mathrm{n}(\%)$ \\
\hline $\begin{array}{l}\text { Instructions on establishing mother's milk supply } \\
\text { Importance of establishing mother's milk supply }\end{array}$ & $3(20)$ \\
$\begin{array}{l}\text { Educational materials or verbal education on breast } \\
\text { milk, breastfeeding, or DHM }\end{array}$ & $11(73)$ \\
$\begin{array}{l}\text { Discharge parents with DHM } \\
\begin{array}{l}\text { Connect parents to DHM bank for postdischarge or } \\
\text { posteligibility use }\end{array}\end{array}$ & $5(33)$ \\
\hline
\end{tabular}

DHM, donor human milk.

parents with DHM (33\%) or connecting parents with a DHM bank to obtain milk independently after discharge or after eligibility period $(40 \%)$. None of the policies explicitly mentioned materials, training, meetings, or other sources of staff education regarding DHM.

\section{Ordering and handling}

Physicians were most often responsible for creating the order for DHM (Table 2). Almost all policies (86\%) mentioned a nonprofit Human Milk Banking Association of North America (HMBANA) milk bank for sourcing DHM. Guidelines for length of time DHM could be stored after thawing were common-60\% require discarding at 24 hours, $33 \%$ at 48 hours. Three (20\%) also specified that DHM would be discarded within 1 hour after being warmed for feeding. Of note, $40 \%(n=6)$ of policies referenced a separate policy for milk handling, which were not evaluated for this analysis.

\section{DHM description}

Less than half of policies discussed the safety of DHM (33\%), and information on pasteurization (20\%) and donor screening (13\%) was uncommon (Table 4). Of these policies, donors were described as "healthy lactating women" who are "carefully screened and tested." Although many policies endorsed human milk as the preferred diet or supplementation for newborns, fewer explicitly stated the preferred supplementation was DHM (53\% and 27\%, respectively). One-third stated health benefits for DHM use, and nearly as many policies mentioned non-nutrient bioactive factors (27\%). Policies mentioned immune properties and growth factors in DHM, and referred to DHM as "the best choice for their baby," and a "source of nutrition and immunities for infants." One policy references the World Health Organization and the American Academy of Pediatrics' support for "the use of PDHM when mother's milk is not available." Nearly all policies $(n=14,93 \%)$ cited references.

\section{Discussion}

To our knowledge, this is the first study to describe the content of policies for DHM use in level 1 newborn care among a group of hospitals. Although DHM in the level 3/4 care setting is supported by evidence of specific health benefits to preterm infants, little is known about the health outcomes of DHM supplementation in the well infant population. Nonetheless, access to DHM is increasingly desired by both families and providers as an alternative to formula supplements. ${ }^{6,14}$ Policies are, therefore, required to guide this practice, particularly because DHM is a relatively limited and expensive 
Table 4. Donor Human Milk Description

n $(\%)$

Safety of DHM source

$5(33)$

Safety of donor screening

$2(13)$

Safety of pasteurization, sterilization

$3(20)$

Endorses donor milk for supplementation

$4(27)$

Endorses human milk as preferred supplementation 8 (53) or diet

Nonnutrient bioactives in DHM

$4(27)$

Health benefits for DHM use

$5(33)$

resource and HMBANA recommends prioritizing for sick preterm infants. ${ }^{16}$ The results of our study provide guidance for hospitals seeking to create or adapt policies for DHM use in the level 1 newborn care setting.

There is significant overlap between our findings and the content categories suggested by the sources used to direct the focus of our analysis; ${ }^{10,11}$ most policies addressed eligibility criteria, parent education, informed consent, and ordering and handling. However, one category suggested by Mother's Milk Bank Northeast-staff education-was entirely absent. We found it notable that most DHM policies did not include language about helping mothers establish their milk supply nor did they emphasize its importance, although this information may be captured in other nursing policies. Most but not all DHM policies considered a mother's intent to provide breast milk in the eligibility criteria, implying-and in some cases, stating explicitly - that DHM should be used as a bridge until mother's milk supply is established. Furthermore, we noted a gap in acknowledging the impact of any type of supplemental milk volume (i.e., DHM or formula) on a mother's ability to establish her milk supply. Similar to the mechanism by which formula supplementation is thought to interfere with long-term lactation success, ${ }^{9,17,18}$ DHM supplementation could also interfere. However, this potential effect of DHM supplementation has received very little study. ${ }^{19}$

We also noted that policies emphasized the benefits of human milk itself, regardless of source (mother versus donor). This emphasis disregards important differences between the content of DHM and mother's own milk due to pasteurization of DHM and number of freeze-thaw cycles, including reduced cytokines, ${ }^{20}$ growth factors, ${ }^{21}$ immune components, ${ }^{22,23}$ and macronutrients such as fat and protein. ${ }^{24}$ Clarifying the superiority of mother's own milk in terms of bioactive and nutritional properties underscores the short-term nature of DHM supplementation and provides an opportunity to emphasize the long-term goal of supporting lactation success. Information on the safety of DHM was described infrequently and may exist within the policies' appendices or attachments excluded from our analysis. Safety information may be more relevant for patient-facing handouts, staff education, and/or separate policies on milk handling.

Recommendations for DHM use are evolving. HBMANA recently released a new set of best practices for expressing, storing, and handling human milk, including updates to the optimal storage times for thawed pasteurized human milk. ${ }^{25}$ The recommendation for discarding milk has now been changed from within 24 hours of thaw to 48 hours, based on evidence of safety. ${ }^{26,27}$ This change reduces cost and waste by decreasing the need to discard thawed milk before it is used. Several policies in this analysis used the 48-hour window, perhaps indicating the anticipation of this update.

Overall, eligibility criteria for DHM supplementation in level 1 care were similar to known criteria for formula supplementation. Eligibility for DHM was in some cases detailed and specific, whereas in others, it was vague and nondescript, and a wide array of criteria were described. Policies containing guidelines for both NICU and well baby DHM use did not always make clear to which population these criteria pertained, nor did they address the differences in levels of evidence for DHM use in different populations.

\section{Recommendations and future research}

One objective of offering DHM in the level 1 population is to provide an all-human milk diet for infants. We suggest that policies explicitly state the importance of facilitating lactation as the long-term goal for DHM use in well-newborn settings. In addition, we recommend including a reference to (and possibly a brief description of) how the level 1 care unit helps mothers establish their milk supply. Outlining the differences between DHM and mother's own milk would further focus the policy on the larger goal of facilitating lactation.

Although our findings indicate the absence of staff education from level 1 DHM policies, we did not collect or analyze data on the presence or quality of staff education. A recent study signals a need for nurse education on DHM use in the level 1 care setting. ${ }^{28}$ Further research is needed to determine gaps in staff education on DHM. Studies on outcomes associated with DHM supplementation in level 1 care are also needed, including those for specific groups such as late preterm infants. ${ }^{29}$

\section{Limitations}

Our study included only a small group of northeast hospitals, additional research is needed to determine the content of level 1 DHM policies in other regions. We did not analyze the appendices included in policy documents, which may have caused relevant details to be omitted and for misclassifications to occur. Our findings may reflect the evolving literature on DHM and the sparse guidance on content for DHM policies.

\section{Conclusion}

Many policies address eligibility for receiving DHM and show how to use DHM in level 1 newborn care. In contrast, few address how to support lactation while DHM is provided, which may be crucial for optimizing long-term breastfeeding outcomes. Standard guidelines for policy content are lacking; based on our findings, we recommend that such guidelines be centered around the goal of promoting and supporting successful lactation rather than simply avoiding formula in level 1 newborn care.

\section{Acknowledgments}

The authors thank Naomi Bar-Yam, PhD, and Cynthia Cohen (Mother's Milk Bank Northeast) for their assistance in identifying professional contacts at the hospitals included in this research. We also thank the hospital staff who provided 
policies for this research. Funding was provided by the Karsh Award, Brigham and Women's Hospital (J.F.R.). This analysis was previously presented at the 23rd Annual International Meeting of the Academy of Breastfeeding Medicine in San Francisco during November 15-17, 2018.

\section{Disclosure Statement}

No competing financial interests exist.

\section{References}

1. Breastfeeding and the use of human milk. Pediatrics 2012; 129:e827-e841.

2. Meier P, Patel A, Esquerra-Zwiers A. Donor human milk update: Evidence, mechanisms, and priorities for research and practice. $J$ Pediatr 2017;180:15-21.

3. Meier P, Patel A, Bigger H, et al. Human milk feedings in the neonatal intensive care unit. In: Diet and Nutrition in Clinical Care, Rajendram R, Preedy V, Patel V, eds. New York: Springer, 2015, pp. 807-822.

4. Committee on Nutrition, Section on Breastfeeding, Committee on Fetus and Newborn. Donor human milk for the high-risk infant: Preparation, safety, and usage options in the United States. Pediatrics 2017;139:e20163440.

5. Parker MG, Barrero-Castillero A, Corwin BK, et al. Pasteurized human donor milk use among US level 3 neonatal intensive care units. J Hum Lact 2013;29:381-389.

6. Belfort MB, Drouin K, Riley JF, et al. Prevalence and trends in donor milk use in the well-baby nursery: A survey of Northeast United States Birth Hospitals. Breastfeed Med 2018;13:34-41.

7. Baby-Friendly USA. Guidelines and Evaluation Criteria for Facilities Seeking Baby-Friendly Designation. Albany, NY: Baby-Friendly USA, 2016.

8. Specifications manual for Joint Commission national quality measures. 2019. Available at https://manual.jointcommission .org/releases/TJC2019A/MIF0170.html (accessed March 28, 2019).

9. Chantry CJ, Dewey KG, Peerson JM, et al. In-hospital formula use increases early breastfeeding cessation among first-time mothers intending to exclusively breastfeed. J Pediatr 2014;164:1339-1345 e1335.

10. Lewis SC, McMahon M, Combs G, et al. The nuts and bolts of implementing a pasteurized donor human milk program on a mother baby unit. J Hum Lact 2018;34:116-119.

11. Implementing a Hospital Donor Milk Program: A Guide for the Use of Pasteurized Donor Human Milk as Standard of Care. Newton Upper Falls, MA: Mothers' Milk Bank Northeast, 2017.

12. AAP Committee on Fetus and Newborn and ACOG Committee on Obstetric Practice. Guidelines for Perinatal Care, 8th ed., 2017.

13. Kair LR, Flaherman VJ. Donor milk or formula: A qualitative study of postpartum mothers of healthy newborns. J Hum Lact 2017;33:710-716.

14. Sen S, Benjamin C, Riley J, et al. Donor milk utilization for healthy infants: Experience at a Single Academic Center. Breastfeed Med 2018;13:28-33.
15. Harris PA, Taylor R, Thielke R, et al. Research electronic data capture (REDCap) — A metadata-driven methodology and workflow process for providing translational research informatics support. J Biomed Inform 2009;42:377-381.

16. HMBANA. Guidelines for the Establishment and Operation of a Donor Human Milk Bank. 10th ed. Fort Worth, TX: HMBANA, 2018.

17. Neville MC. Anatomy and physiology of lactation. Pediatr Clin North Am 2001;48:13-34.

18. DiGirolamo AM, Grummer-Strawn LM, Fein SB. Effect of maternity-care practices on breastfeeding. Pediatrics 2008; 122 Suppl 2:S43-S49.

19. Kair LR, Flaherman VJ, Colaizy TT. Effect of donor milk supplementation on breastfeeding outcomes in term newborns: A randomized controlled trial. Clin Pediatr 2019;58: 534-540.

20. Untalan P, Keeney S, Palkowetz K, et al. Heat susceptibility of interleukin-10 and other cytokines in donor human milk. Breastfeed Med 2009;4:137-144.

21. Goelz R, Hihn E, Hamprecht K, et al. Effects of different CMV-heat-inactivation-methods on growth factors in human breast milk. Pediatr Res 2009;65:458-461.

22. Ewaschuk JB, Unger S, O'Connor DL, et al. Effect of pasteurization on selected immune components of donated human breast milk. J Perinatol 2011;31:593-598.

23. Akinbi H, Meinzen-Derr J, Auer C, et al. Alterations in the host defense properties of human milk following prolonged storage or pasteurization. J Pediatr Gastroenterol Nutr 2010;51:347-352.

24. Vieira AA, Soares FV, Pimenta HP, et al. Analysis of the influence of pasteurization, freezing/thawing, and offer processes on human milk's macronutrient concentrations. Early Hum Dev 2011;87:577-580.

25. Jones F. Best Practice for Expressing, Storing, and Handling Human Milk in Hospitals, Homes, and Child Care Settings. 4th ed. Fort Worth, TX: Human Milk Banking Association of North America, 2019.

26. Vickers A, Starks-Solis S, Hill D, et al. Pasteurized donor human milk maintains microbiological purity for 4 days at 4 degrees C. J Hum Lact 2015;31:401-405.

27. Meng T, Perrin M, Allen J, et al. Storage of unfed and leftover pasteurized human milk. Breastfeed Med 2016;11: 538-543.

28. Ferrarello D, Froh EB, Hinson TD, et al. Nurses' views on using pasteurized donor human milk for hypoglycemic term infants. MCN Am J Matern Child Nurs 2019;44:157-163.

29. Mannel R, Peck JD. Outcomes associated with type of milk supplementation among late preterm infants. J Obstet Gynecol Neonatal Nurs 2018;47:571-582.

Address correspondence to: Kaitlin H. Drouin, MS, MA Department of Pediatric Newborn Medicine Brigham and Women's Hospital 221 Longwood Avenue, 3rd Floor Boston, MA 02115

E-mail: kdrouin@bwh.harvard.edu 